\title{
A CHARACTERISTIC PROPERTY OF MINIMAL SURFACES*
}

\author{
BY JESSE DOUGLAS $†$
}

1. Statement of Theorems. By the mean curvature of a surface at a point we shall understand $1 / R_{1}+1 / R_{2}$, where $1 / R_{1}, 1 / R_{2}$ are the principal curvatures at the point. There is some divergence in the literature with respect to this nomenclature; some authors call $\left(1 / R_{1}+1 / R_{2}\right) / 2$ the mean curvature. $\ddagger$

Imagine that about any point $O$ of an arbitrary surface $\Sigma$ as center we describe a sphere $S$ of infinitesimal radius $r$. Then $S$ is intersected by $\Sigma$ in a curve $C$ infinitely close $\S$ to the great circle cut from $S$ by the tangent plane to $\Sigma$ at $O$. The curve $C$ divides the surface of $S$ into two areas nearly equal to each other. Also, the portion of $\Sigma$ lying within $S$ divides $S$ into two nearly equal volumes.

The present paper is devoted to the proofs of the following three theorems.

TheOREM I. Let the difference between the two areas into which $C$ divides $S$ be denoted by $\eta$. Then, in the limit as $r \rightarrow 0$,

$$
\frac{\eta}{V}=\frac{3}{4}\left(\frac{1}{R_{1}}+\frac{1}{R_{2}}\right)
$$

where $V$ denotes the volume of $S$.

THEOREM II. Let $v$ denote the difference of the two volumes into which $S$ is divided by $\Sigma$. Then, in the limit as $r \rightarrow 0$,

* Presented to the Society, October 30, 1926.

$\dagger$ National Research Fellow in Mathematics.

$¥$ Bianchi, Eisenhart, and Scheffers use the first notation; Blaschke and Darboux the second.

$\S$ Even in comparison with the radius of the sphere, that is, the deviation of $C$ from the great circle is an infinitesimal of the second or higher order. 


$$
\frac{v}{r V}=\frac{3}{16}\left(\frac{1}{R_{1}}+\frac{1}{R_{2}}\right) .
$$

When $\Sigma$ is a minimal surface, or one of mean curvature zero, these theorems yield the following corollary.

Theorem III. A minimal surface bisects the area and volume of an infinitesimal sphere about any point of the surface as center.

The sense of this statement is that the bisection occurs to infinitesimals of higher order for a minimal surface than for a non-minimal surface.

2. Proof of Theorem I. The proof of these theorems is a straightforward problem in calculus. We choose for axes the tangents to the lines of curvature at $O$ and the normal to the surface. Then, for all our purposes, the surface may be replaced by the paraboloid

$$
z=a x^{2}+b y^{2}
$$

having second order contact with it at $O$. Here

$$
a=\frac{1}{2 R_{1}}, \quad b=\frac{1}{2 R_{2}} .
$$

The equation of the sphere $S$ is

$$
x^{2}+y^{2}+z^{2}=r^{2} .
$$

We use cylindrical coordinates $\rho, \theta, z$, in terms of which the equations of $\Sigma, S$ are respectively

$$
\begin{gathered}
z=\rho^{2}\left(a \cos ^{2} \theta+b \sin ^{2} \theta\right), \\
\rho^{2}+z^{2}=r^{2} .
\end{gathered}
$$

The polar equation of the projection $C_{1}$ of $C$ on the tangent plane at $O$ is

$$
\rho_{1}^{2}+\rho_{1}{ }^{4}\left(a \cos ^{2} \theta+b \sin ^{2} \theta\right)^{2}=r^{2} .
$$

Expanding $\rho_{1}$ in powers of $r$, we need retain only the first two terms, and have

$$
\rho_{1}=r-\frac{1}{2} r^{3}\left(a \cos ^{2} \theta+b \sin ^{2} \theta\right)^{2}
$$


for the (approximate) polar equation of $C_{1}$. The area of the portion of the sphere above $C$ is

$$
A_{1}=4 \int_{0}^{\pi / 2} \int_{0}^{\rho_{1}} \frac{r}{\left(r^{2}-\rho^{2}\right)^{1 / 2}} \rho d \rho d \theta .
$$

Performing the first integration, we get

$$
A_{1}=4 r \int_{0}^{\pi / 2}\left\{-\left(r^{2}-\rho^{2}\right)^{1 / 2}\right\}_{0}^{\rho_{1}} d \theta .
$$

Now we have

$$
\left\{-\left(r^{2}-\rho^{2}\right)^{1 / 2}\right\}_{0}^{\rho_{1}}=r-r^{2}\left(a \cos ^{2} \theta+b \sin ^{2} \theta\right)
$$

where, as will be our rule hereafter, we neglect all infinitesimals beyond those which would influence the final result. Performing the second integration we find

$$
A_{1}=2 \pi r^{2}-\pi r^{3}(a+b) .
$$

The area of the portion of the sphere to the other side of $C$ is the complement of $A_{1}$ as to $4 \pi r^{2}$, or

$$
A_{2}=2 \pi r^{2}+\pi r^{3}(a+b) .
$$

The difference

$$
\eta=A_{2}-A_{1}=2 \pi r^{3}(a+b)=\pi r^{3}\left(\frac{1}{R_{1}}+\frac{1}{R_{2}}\right)
$$

by (2). Introducing $V=\frac{4}{3} \pi r^{3}$, we have

or Theorem I.

$$
\frac{\eta}{V}=\frac{3}{4}\left(\frac{1}{R_{1}}+\frac{1}{R_{2}}\right)
$$

3. Proof of Theorem II. We first obtain the volume $V_{1}$ above $\Sigma$ and below the sphere by calculating the difference $V_{1}=V_{S}-V_{\Sigma}$, where

$$
\begin{aligned}
& V_{S}=\text { volume under portion of sphere above } C, \\
& V_{\Sigma}=\text { volume under portion of } \Sigma \text { within } C .
\end{aligned}
$$


The volume $V_{S}$ turns out to be that of a hemisphere up to infinitesimals of the sixth order. For, with the use of (5) and (6),

$$
\begin{aligned}
V_{S} & =4 \int_{0}^{\pi / 2} \int_{0}^{\rho_{1}}\left(r^{2}-\rho^{2}\right)^{1 / 2} \rho d \rho d \theta \\
& =4 \int_{0}^{\pi / 2} \frac{1}{3}\left\{r^{3}-\left(r^{2}-\rho_{1}^{2}\right)^{3 / 2}\right\} d \theta \\
& =\frac{4}{3} \int_{0}^{\pi / 2}\left\{r^{3}-r^{6}\left(a \cos ^{2} \theta+b \sin ^{2} \theta\right)^{3}\right\} d \theta=\frac{2}{3} \pi r^{3},
\end{aligned}
$$

neglecting the term in $r^{6}$.

$$
V_{\Sigma}=\iint_{\Sigma_{1}} z \rho d \rho d \theta
$$

where $\Sigma_{1}$ is the area enclosed by $C_{1}$, the projection of $C$. By (4),

$$
\begin{aligned}
V_{\Sigma} & =4 \int_{0}^{\pi / 2} \int_{0}^{\rho_{1}} \rho^{3}\left(a \cos ^{2} \theta+b \sin ^{2} \theta\right) d \rho d \theta \\
& =\int_{0}^{\pi / 2} \rho_{1}{ }^{4}\left(a \cos ^{2} \theta+b \sin ^{2} \theta\right) d \theta .
\end{aligned}
$$

By (6), $\rho_{1}^{4}=r^{4}$ up to terms in $r^{6}$ and higher powers. Therefore $V_{\Sigma} \pi r^{4}(a+b) / 4$. It follows that

$$
V_{1}=\frac{2}{3} \pi r^{3}-\frac{\pi}{4} r^{4}(a+b) .
$$

The complementary volume of $V_{1}$ as to the sphere is

$$
V_{2}=\frac{2}{3} \pi r^{3}+\frac{\pi}{4} r^{4}(a+b) .
$$

Dividing the difference $v=V_{2}-V_{1}$ by $r V=4 \pi r^{4} / 3$, we obtain

$$
\frac{v}{r V}=\frac{3}{8}(a+b)=\frac{3}{16}\left(\frac{1}{R_{1}}+\frac{1}{R_{2}}\right)
$$

that is, Theorem II.

Princeton University 